\title{
Recrû naturel dans des taillis de chêne vert (Quercus ilex L) à la suite d'exploitations partielles
}

\author{
M Ducrey *, M Boisserie
}

INRA, station de sylviculture méditerranéenne, avenue A Vivaldi, 84000 Avignon, France

(Reçu le 3 mai 1991; accepté le 13 décembre 1991)

\begin{abstract}
Résumé - L'objectif de ce travail est d'étudier l'influence de l'âge du taillis (4-57 ans) et de l'intensité du dépressage (fort ou faible) ou de l'éclaircie (26-78\% de surface terrière enlevée) sur l'apparition et la croissance des nouveaux rejets de souche dans des taillis de chêne vert.

L'ensemble des rejets de chêne vert et autres espèces arbustives constitue une strate quantifiée par le taux de recouvrement, la biomasse et la hauteur des rejets de chêne vert. Cette strate s'accroît régulièrement pendant les 5 années suivant la coupe et augmente avec l'intensité d'éclaircie. Par contre, elle est plus importante dans les taillis jeunes ( 25 ans et moins) que dans les taillis âgés.

La réaction du taillis est ensuite étudiée au niveau de la cépée. Le nombre et la croissance des rejets apparus dans chaque cépée sont alors reliés à l'état initial et au degré d'exploitation de la cépée. Le nombre de rejets est proportionnel au nombre de brins coupés dans la cépée tandis que leur croissance est plutôt liée au pourcentage de brins ou de surface terrière enlevé en éclaircie. On cherche enfin à expliquer le fonctionnement de la cépée en séparant l'effet du niveau moyen de l'éclaircie dans la placette et du degré de coupe dans la cépée. En conclusion, des hypothèses sont formulées sur l'origine physiologique aes phénomènes observés.
\end{abstract}

Quercus ilex $=$ chêne vert $/$ taillis / éclaircle / cépée / rejet de souche

Summary - Natural regrowth of holm oak coppice (Quercus ilex $\mathrm{L}$ ) following partial cuts. The aim of this article was to study the influence of coppice age (4 to 57 years old) and of intensities of cleaning (light and heavy) or thinning (26-78\% of removed basal area) on the appearance and growth of new stump sprouts in Quercus ilex coppice. The sprout layer at soil level can be quantified by degree of closure, biomass and Quercus ilex sprout height. This layer grew regularly for the 5 years following harvest and increased in direct proportion to increase in thinning intensities; it was found to be greater for young coppice (25 years old and less) than for old coppice. Coppice functioning was then studied at the stool level. Number and growth of new sprouts inside each stool were then related to the initial characteristics of the initial stool as well as to the degree of thinning in the stool. Sprout number was proportional to number of cut shoots, while sprout growth tended to be related to percentage of shoot or basal area removed by thinning. Finally an attempt has been made to explain the functioning of the stools by separating the effects of the mean thinning intensity of each plot and of the degree of cutting for each stool. In conclusion, hypotheses have been proposed on physiological origin of the observed phenomena.

Quercus ilex = holm oak / coppice $/$ thinning / stool / stump sprout

* Correspondance et tirés à part 


\section{INTRODUCTION}

Les taillis de chêne vert ont actuellement comme principal débouché économique la production de bois de chauffage. Dans ce but, ils sont exploités par coupe rase tous les 20 ou 30 ans et ne sont l'objet d'aucune intervention entre 2 coupes. Mais actuellement on est tenté de les valoriser différemment (Ducrey, 1988).

II s'agit essentiellement de dépressages des jeunes taillis ou d'éclaircies dans les taillis plus âgés, réalisés dans le but de relever le couvert pour le rendre plus défensable contre le feu ou dans le but d'entamer une conversion en futaie sur souche.

II peut aussi s'agir de balivage (sélection d'un nombre limité de brins, théoriquement de franc-pied, mais le plus souvent le plus beau brin dans une cépée) pour réaliser des pare-feu arborés en bordure de routes ou de zones urbaines ou encore pour une utilisation sylvopastorale.

Ces opérations sylvicoles sont inhabituelles dans le cas des taillis de chêne vert où, traditionnellement, il n'y a aucune intervention entre 2 coupes à blanc (Jolyet, 1916; Mathey, 1929; Perrin, 1964; Seigue, 1985). Aussi, nous manquons actuellement de connaissances scientifiques à leur sujet. Elles sont aussi assez peu fréquentes pour les autres espèces forestières, les seules exceptions s'apparentant au "furetage" (exploitation partielle du taillis à des intervalles de temps rapprochés conduisant à des cépées composées de brins d'âges différents) technique maintenant très peu employée (Perrin, 1964).

Nous avons étudié au cours d'un précédent article (Ducrey et Toth, 1992) l'influence de l'intensité du dépressage ou de l'éclaircie sur la croissance des brins du taillis laissés sur pied et mis en évidence l'effet positif de ces opérations sur la croissance en circonférence quand on enlève plus de $50 \%$ de la surface terrière initiale.
Par contre, les éclaircies ont un effet nul, voire négatif sur la croissance en hauteur.

Dans un taillis, l'entité biologique est la cépée constituée de plusieurs brins partant de la même souche (Auclair, 1986). Lors d'une coupe à blanc, ou même lorsqu'on coupe seulement quelques brins dans une cépée, il y a apparition de nouveaux rejets à partir de bourgeons situés à la base des brins coupés et sur la partie voisine de la souche. L'origine des bourgeons qui produisent ces rejets a fait l'objet de nombreuses études (Bartet, 1890; Kormanik et Brown, †973; Fink, 1983; Kauppi et al, 1987), mais aucune ne s'applique directement au chêne vert.

Ainsi, lors d'une éclaircie dans un taillis, on favorise la croissance des brins restants, mais on déclenche aussi l'apparition de nouveaux rejets de souche. La présence de ces rejets vient souvent contrecarrer le but recherché, notamment dans le cas de la conversion en futaie sur souche. II faut donc éliminer ces nouveaux rejets. On peut aussi tenter de contrôler leur apparition et leur croissance en dosant convenablement l'intensité des opérations sylvicoles (Piskoric, 1963; Weinstein, 1986).

Le but de cet article est d'étudier la dynamique d'apparition et de croissance des rejets de souche dans des taillis de chêne vert de différents âges (4-57 ans) et qui ont fait l'objet de dépressages ou d'éclaircies d'intensité variable.

\section{MATÉRIEL ET MÉTHODES}

\section{Le dispositif expérimental}

Le dispositif expérimental utilisé a été décrit en détail dans un article antérieur (Ducrey et Toth, 1992). Aussi, nous ne rappellerons que ce qui est strictement nécessaire à la compréhension de cet article. 


\section{Cas des jeunes taillis de La Bruguière}

L'étude du dépressage dans des taillis âgés de 4 à 25 ans est faite en forêt communale de La Bruguière dans le Gard (Ducrey et al, 1986). Dépressage est le terme consacré pour l'éclaircie des jeunes peuplements : il s'agit de desserrer les jeunes brins de taillis pour augmenter leur espace disponible, sans objectif a priori de sélection. On a étudié 3 traitements sylvicoles : dépressage fort, dépressage faible et témoin non dépressé. Ces traitements ont été répétés 2 fois dans chacune des 5 classes d'âge étudiées : 4 , $8,15,20$ et 25 ans fin 1984. Le dispositif expérimental est donc constitué de 30 placettes de 9 a chacune dans lesquelles on étudie plus particulièrement l'are central : recouvrement du sol, croissance et biomasse des rejets.

\section{Cas des vieux taillis de Puéchabon}

L'étude des éclaircies dans des taillis âgés de 43 et 57 ans, fin 1985, est faite en forêt domaniale de Puéchabon dans l'Hérault (Ducrey et al, 1987). On étudie plus particulièrement l'effet de l'intensité de l'éclaircie. II y a au total 5 traitements : éclaircies faible, moyenne, forte et très forte ayant enlevé respectivement $26,42,58$ et $78 \%$ de la surface terrière initiale et témoin non éclairci. Les traitements ont été installés 2 fois dans le taillis de 43 ans (blocs 1 et 2 ) et une fois dans le taillis de 57 ans (bloc 3). La taille des placettes élémentaires varie de 10 à 12 a. En raison de la similitude de comportement des taillis de 43 et 57 ans, on a privilégié, dans les résultats, l'analyse des différences entre blocs. Dans ce dispositif, on étudie plus particulièrement le recouvrement du sol et la dynamique des rejets en relation avec les caractéristiques des cépées auxquelles ils appartiennent.

Les caractéristiques dendrométriques des taillis étudiés sont données dans le tableau I.

Rappelons aussi qu'il y a équivalence, du point de vue de la densité après éclaircie, entre le dépressage faible et l'éclaircie forte d'une part, le dépressage fort et l'éclaircie très forte d'autre part. Indiquons enfin que, pour la clarté de l'article, les taillis seront toujours désignés par l'âge qu'ils avaient au moment de l'installation des dispositifs expérimentaux.

\section{Les mesures de recouvrement}

La couverture ligneuse arbustive constituée des rejets de chêne vert et des rejets des autres arbustes recépés au moment de la coupe a été inventoriée sur l'are central de chaque placette dépressée ou éclaircie dans chacun des 2 dispositifs de La Bruguière et de Puéchabon. Ces observations n'ont pas été faites dans les témoins où il n'y a pas de nouveaux rejets de

Tableau I. Caractéristiques dendrométriques moyennes des taillis étudiés. Pour La Bruguière et Puéchabon, les mesures et comptages ont été réalisés respectivement fin 1984 et fin 1985 sur tous les brins dont la circonférence à $0,50 \mathrm{~m}$ est supérieure à $3 \mathrm{~cm}$. Dans tous les cas, on a mesuré la hauteur du plus haut brin de la cépée.

\begin{tabular}{|c|c|c|c|c|c|}
\hline Forêt & $\hat{A} g e$ & $\begin{array}{l}\text { Nombre } \\
\text { de brins } \\
\text { (N/ha) }\end{array}$ & $\begin{array}{l}\text { Circonférence } \\
\text { moyenne } \\
(\mathrm{cm})\end{array}$ & $\begin{array}{l}\text { Surface } \\
\text { terrière } \\
\left(m^{2} / h a\right)\end{array}$ & $\begin{array}{l}\text { Hauteur } \\
\text { moyenne } \\
(m)\end{array}$ \\
\hline \multirow{5}{*}{ La Bruguière } & 4 & 29900 & 4,3 & 4,68 & 1,66 \\
\hline & 8 & 30300 & 5,9 & 10,37 & 2,10 \\
\hline & 15 & 31000 & 7,5 & 16,84 & 2,56 \\
\hline & 20 & 17800 & 12,6 & 28,63 & 3,72 \\
\hline & 25 & $12<00$ & 14,9 & 27,20 & 3,75 \\
\hline & 43 & 8700 & 21,3 & 31,5 & 4,73 \\
\hline Puéchabon & 57 & 6300 & 28,2 & 41,6 & 6,14 \\
\hline
\end{tabular}


souche. Le recouvrement est mesuré par un échantillon de 200 points répartis tous les 50 $\mathrm{cm}$ le long de 10 lignes parallèles espacées de $1 \mathrm{~m}$. Pour chaque point, on a noté l'espèce présente et la hauteur du rejet quand il s'agissait de chêne vert. $\grave{A}$ La Bruguière, les principales espèces répertoriées sont : Quercus ilex $L$, Phillyrea sp, Ligustrum vulgare $L$ et Prunus spinosa L. A Puéchabon, il s'agit de Quercus ilex L, Phillyrea sp, Buxus sempervirens L, Pistacia sp et Ruscus aculeatus $L$.

Ces données ont permis de calculer pour chaque ligne et pour chaque placette les pourcentages de recouvrement en chêne vert, en espéces diverses et total. Pour les rejets de chêne vert, on a aussi calculé les hauteurs moyennes (calculées sur les rejets présents) et corrigées (calculées sur l'ensemble des points d'échantillonnage) pour tenir compte des vides.

\section{Les mesures de biomasse}

Elles concernent la moitié des placettes dépressées du dispositif de La Bruguière, soit 10 placettes au total. L'are central a été divisé en 10 bandes correspondant aux lignes déchantillonnage du recouvrement. Sur chaque ligne, toute la végétation ayant poussé depuis le dépressage initial a été coupée puis pesée en séparant les différentes espèces. Des échantillons ont été récoltés pour calculer le taux d'humidité après passage à l'étuve à $65^{\circ} \mathrm{C}$ jusqu'à obtention d'un poids constant. La séparation des feuilles et des tiges des échantillons de chêne vert a permis de calculer le pourcentage de biomasse foliaire.

On dispose donc, pour chaque ligne d'échantillonnage et pour l'ensemble de chaque placette centrale de $1 \mathrm{a}$, de la biomasse des feuilles et des tiges de chêne vert, ainsi que de la biomasse totale des autres espèces présentes.

\section{Caractérisation du recrû au niveau des cépées}

Dans le dispositif de Puéchabon, le recrû a été caractérisé par le taux de recouvrement en chêne vert, mais aussi par les rejets qui sont apparus dans chaque cépée.
Dans chacune des 15 placettes de ce dispositif expérimental, 50 cépées ont été choisies : les 45 cépées auxquelles appartiennent les arbres échantillons utilisés pour l'étude de croissance du taillis (dans ces cépées la coupe est partielle, plus rarement nulle) et 5 cépées entièrement coupées. On a ainsi une répartition des cépées telle que toutes les classes de pourcentage de surface terrière coupée par cépée sont représentées.

Dans chacune de ces cépées, nous avons relevé, pour chaque brin coupé, le nombre de rejets s'y rattachant et la hauteur du plus grand de ces rejets. Nous avons aussi déterminé les caractéristiques de ces cépées juste après l'éclaircie fin 1985.

Chaque cépée a été caractérisée par 10 variables :

- le nombre de brins coupés (B_COUPES);

- le nombre total de brins avant la coupe (B_TOTAL);

- le pourcentage de brins coupés (PC_B_CP);

- la circonférence moyenne (à 1,30 m) des brins restant sur pied (CM_BRIN);

- la circonférence moyenne des brins coupés (CM_B_CP);

- la circonférence moyenne de tous les brins (CM_B_TOT);

- la surface terrière des brins restant sur pied (G_BRIN);

- la surface terrière des brins coupés (G_B_CP);

- la surface terrière de tous les brins de la cépée initiale (G_TOT);

- le pourcentage de surface terrière enlevée en éclaircie (PC_G_CP).

Les nouveaux rejets apparus dans une cépée à la suite de l'éclaircie sont caractérisés par 7 variables :

- le nombre total de rejets;

- le nombre moyen de rejets par brin coupé;

- le nombre moyen de rejets par pourcent de brins coupés;

- le nombre de rejets par unité de surface terrière enlevée;

- le nombre de rejets par pourcent de surface terrière enlevée;

- la hauteur moyenne des rejets dominants de chaque brin coupé;

- la hauteur du rejet le plus grand. 
Les observations ont été faites 1 an et 4 ans après l'éclaircie.

\section{RÉSULTATS}

\section{Recouvrement du sol par les rejets}

Les résultats des observations de recouvrement faites 1 an, 2 ans et 5 ans après dépressage des taillis jeunes de La Bruguière sont donnés sur la figure 1 et ceux des observations faites 1 an et 4 ans après éclaircie des taillis âgés de Puéchabon sur la figure 2.

On remarque tout d'abord une augmentation au cours du temps du taux de recouvrement en chêne vert et en espèces diverses pour l'ensemble des 2 dispositifs. Ainsi le taux moyen de recouvrement en chêne vert passe de $23,4 \%$ en 1985 à $30,1 \%$ en 1986 et $44,4 \%$ en 1989 à $\mathrm{La}$ Bruguière et de $12,4 \%$ en 1986 à $34,5 \%$ en 1989 pour Puéchabon. Le recouvrement en espèces diverses augmente dans les mêmes proportions, aussi les pourcentages de vides diminuent régulièrement et ne sont plus que de $15,5 \%$ à La Bruguière et $45,1 \%$ à Puéchabon en 1989.

L'âge du taillis ne paraît pas avoir d'influence marquée sur le taux de recouvrement. Les différences constatées semblent davantage liées à la fertilité des stations ou à leur composition floristique plutôt qu'à un effet de l'âge du taillis.

L'effet de l'intensité du dépressage ou de l'éclaircie est particulièrement intéressant à étudier.

II y a globalement peu de différences entre le dépressage faible et le dépressage fort pour le recouvrement total. Par contre, des différences apparaissent la $5^{\mathrm{e}}$ année pour le taux de chêne vert qui est généralement plus élevé dans le dépressage fort que dans le dépressage faible.
Mais il y a compensation avec les espèces diverses aussi le recouvrement total n'est-il pas différent!

Pour les taillis âgés, pour lesquels on dispose d'une gamme d'éclaircies plus importante, on voit qu'il n'y a, en 1989, pratiquement pas de différences entre les éclaircies faibles et moyennes : environ $23 \%$ de chêne vert et $46 \%$ de recouvrement total, alors que ces pourcentages augmentent significativement pour atteindre respectivement $38 \%$ et $59 \%$ pour l'éclaircie forte et finalement $53 \%$ et $69 \%$ pour l'éclaircie très forte.

\section{Biomasse des rejets}

La biomasse des rejets a été étudiée dans les 10 placettes recépées, sur une surface de 1 a divisée en 10 bandes de $10 \mathrm{~m} \times 1$ $m$. Ceci permet d'effectuer une analyse de variance à 2 facteurs (âge et traitement) à partir des données de chaque bande (tableau II).

La biomasse de chêne vert est significativement plus élevée dans les dépressages forts $(6,0$ tha) que dans les dépressages faibles (3,0 tha). L'effet de l'âge se traduit par une augmentation constante de la biomasse de chêne vert (de 3,8 tha à 4 ans à 5,3 t/ha à 25 ans), mais cet effet n'est pas significatif. Les résultats obtenus pour la seule biomasse foliaire de chêne vert vont dans le même sens.

La proportion de biomasse foliaire par rapport à la biomasse totale de chêne vert a aussi été calculée. Elle est relativement constante pour les différents âges mais elle est toujours plus élevée pour les dépressages faibles $(37 \%)$ que pour les dépressages forts $(32 \%)$ bien que la biomasse foliaire soit toujours plus faible dans les dépressages faibles que dans les dépressages forts. 


\section{9}
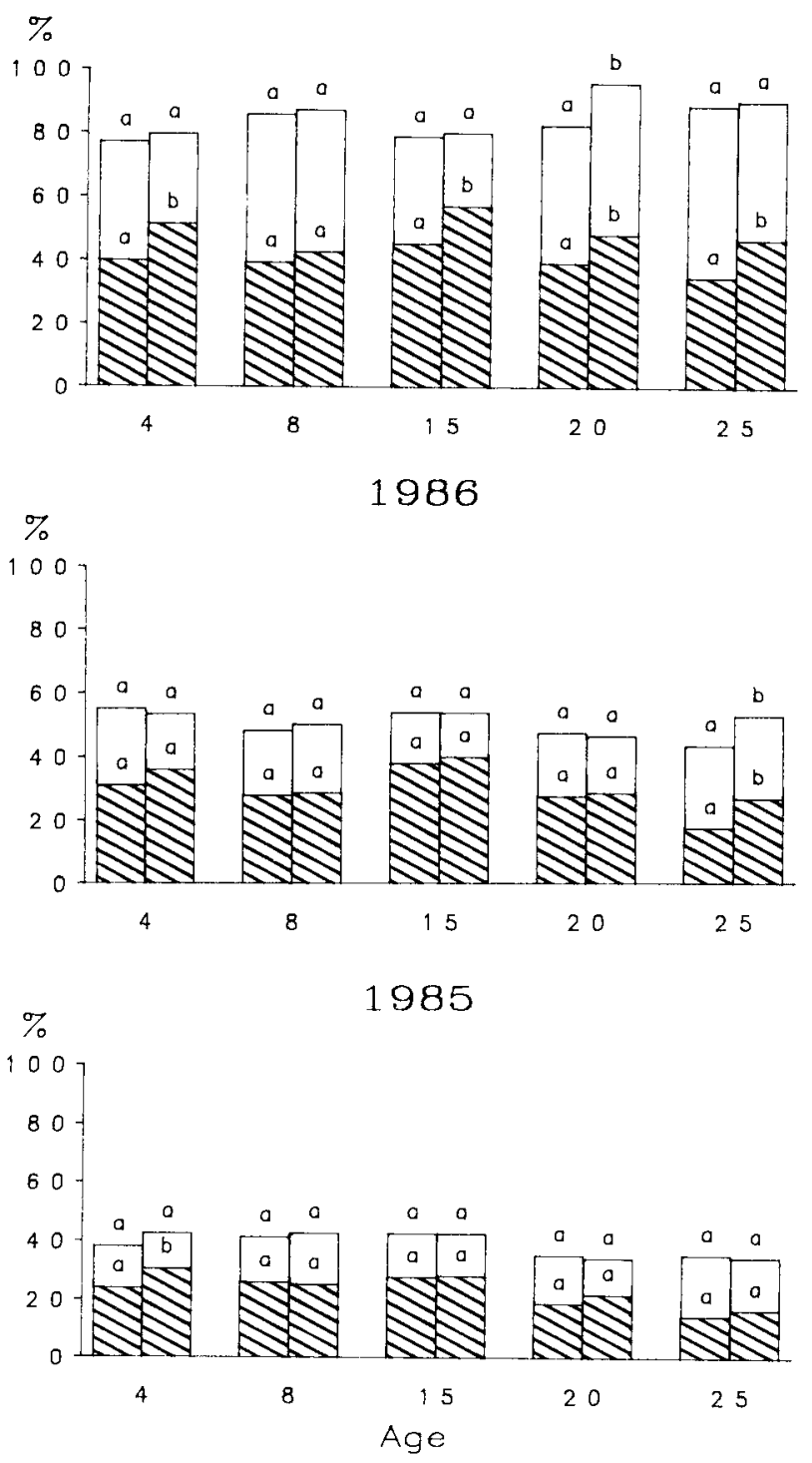

Fig 1.Taux de recouvrement du sol en 1985, 1986 et 1989 dans les taillis jeunes faiblement ou fortement dépressés de la Bruguière. Pour chaque âge : la barre de gauche correspond au dépressage faible, celle de droite au dépressage fort; la partie hachurée concerne le recouvrement en chêne vert, celle non hachurée le recouvrement en espèces diverses; les lettres du haut indiquent, si elles sont différentes, des taux de recouvrement total statistiquement différents au seuil de $5 \%$ (test $\chi^{2}$ de comparaison de pourcentages) et celles du bas la même chose pour le taux de recouvrement en chêne vert. 


\section{9}

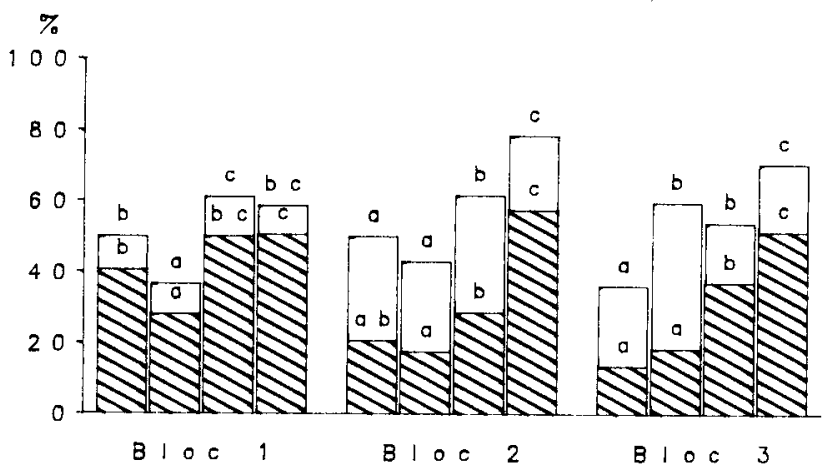

1986

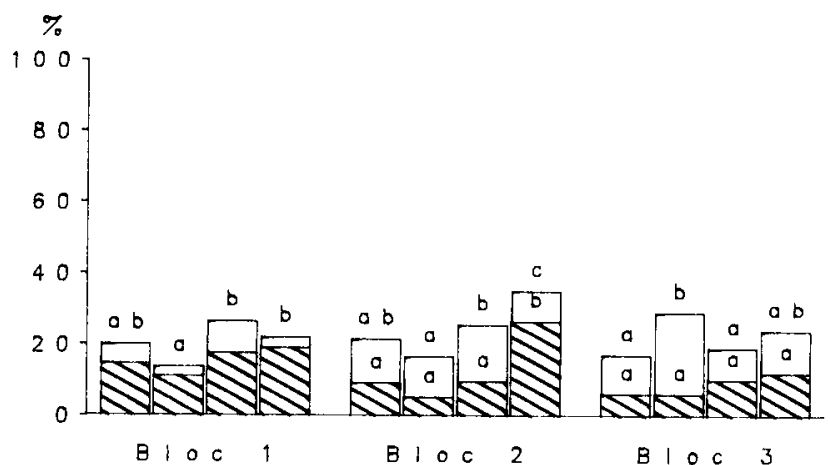

Fig 2. Taux de recouvrement du sol en 1986 et 1989 dans les taillis âgés faiblement, moyennement, fortement ou très fortement éclaircis de Puéchabon. Pour chaque bloc : les barres correspondent, de gauche à droite, aux éclaircies faible, moyenne, forte et très forte; la partie hachurée concerne le recouvrement en chêne vert, celle non hachurée le recouvrement en espèces diverses; les lettres du haut indiquent, si elles sont différentes, des taux de recouvrement total statistiquement différents au seuil de $5 \%$ (test $\chi^{2}$ de comparaison de pourcentages) et celles du bas la même chose pour le taux de recouvrement en chêne vert.

La biomasse totale des rejets, 5 ans après dépressage est en moyenne de 5,0 tha pour le dépressage faible et de $8,5 \mathrm{t}$ ha pour le dépressage fort avec une amplitude de 3,6 tha (dépressage faible, 4 ans) à 10,0 t/ha (dépressage fort, 25 ans).
Nous avons aussi cherché à expliquer la biomasse de chêne vert en fonction des variables caractérisant le recouvrement en chêne vert, données plus faciles à obtenir que la biomasse. Ces variables : taux de recouvrement (PCENTCV), hauteur 
Tableau II. Biomasse foliaire et totale de chêne vert et biomasse totale des rejets; moyennes par âge et par traitement. Résultats de l'analyse de variance. (Des moyennes reliées par un trait ne sont pas statistiquement différentes au seuil de $5 \%$ ). Les effets âge ou traitement ou l'interaction sont significatifs au seuil de $5 \%\left({ }^{*}\right)$, significatifs au seuil de $1 \%\left(^{* *}\right)$, ou non significatifs au seuil de $5 \%$ (NS).

\begin{tabular}{|c|c|c|c|}
\hline \multirow{2}{*}{$\begin{array}{l}\text { Biomasse moyenne } \\
\text { (tha) }\end{array}$} & \multicolumn{2}{|c|}{ Chêne vert } & \multirow{2}{*}{$\begin{array}{l}\text { Biomasse } \\
\text { totale }\end{array}$} \\
\hline & $\begin{array}{l}\text { Biomasse } \\
\text { foliaire }\end{array}$ & $\begin{array}{l}\text { Biomasse } \\
\text { totale }\end{array}$ & \\
\hline \multicolumn{4}{|l|}{ Moyenne par âge } \\
\hline 4 ans & $1,228^{a}$ & $3,755^{a}$ & $5,576^{a}$ \\
\hline 8 ans & $1,208^{a}$ & $3,914^{a}$ & $6,557^{\mathrm{ab}}$ \\
\hline 15 ans & $1,550^{\mathrm{ab}}$ & $4,319^{a}$ & $6,409^{a b}$ \\
\hline 20 ans & $1,681^{a b}$ & $5,253^{a}$ & 7,3396 \\
\hline 25 ans & $1,937^{b}$ & $5,287^{a}$ & $7,853^{b}$ \\
\hline \multicolumn{4}{|c|}{ Moyenne par traitement } \\
\hline Dépressage faible & $1,101^{a}$ & $2,983^{a}$ & $5,041^{a}$ \\
\hline Dépressage fort & $1,941^{b}$ & $6,029 b$ & $8,453^{b}$ \\
\hline $\begin{array}{l}\text { Effet âge } \\
\text { Effet traitement } \\
\text { Interaction }\end{array}$ & $\begin{array}{l}F=3,05\left(^{*}\right) \\
F=28,0\left(^{(*)}\right) \\
F=2,03(\mathrm{NS})\end{array}$ & $\begin{array}{l}F=1,82(\mathrm{NS}) \\
F=39,8\left(^{(* *}\right) \\
F=1,49 \text { (NS) }\end{array}$ & $\begin{array}{l}F=2,61{\left(\left(^{*}\right)\right.}=49,0\left(^{\star *}\right) \\
F=1,59(\mathrm{NS})\end{array}$ \\
\hline
\end{tabular}

moyenne (HAUTMOY) et hauteur corrigée (HAUTCOR) des rejets de chêne vert, sont définies pour chacune des 10 lignes de mesure de chaque placette.

L'analyse des coefficients de corrélations partielles entre la biomasse et les variables explicatives montre (tableau III) que la hauteur corrigée a le plus fort coefficient de corrélation dans 7 placettes sur 10. Ceci peut s'expliquer par le fait que la hauteur corrigée tient compte des vides, ce que ne fait pas la hauteur moyenne. Le regroupement des placettes de même âge ou ayant subi le même type de dépressage donne de moins bons résultats car on regroupe des rejets ayant poussé dans des conditions expérimentales différentes, et donc, ayant des morphologies différentes.

\section{Réaction des cépées}

La réaction des cépées à l'éclaircie, c'està-dire la production de nouveaux rejets, est analysée à 2 niveaux : tout d'abord au niveau de l'intensité globale de l'éclaircie dans la placette, puis au niveau du taux d'exploitation de chaque cépée. Cette étude est faite dans les taillis de Puéchabon âgés de 43 et 57 ans.

La figure 3 résume les principaux résultats concernant l'effet de l'intensité d'éclaircie sur l'apparition et la croissance des rejets dans les cépées, 1 année et 4 années après l'éclaircie.

On note tout d'abord un effet positif et significatif de l'intensité d'éclaircie sur le nombre de rejets par cépée. On voit aussi 
Tableau III. Coefficients de corrélations partielles ( $r$ ) entre la biomasse des rejets de chêne vert et les variables caractérisant le recouvrement : hauteur moyenne (HAUTMOY), hauteur corrigée (HAUTCOR) et taux de recouvrement (PCENTCV). Les coefficients de corrélation apparaissant en italique indiquent la variable qui, pour chaque cas étudié, explique le mieux la biomasse des rejets de chêne vert.

\begin{tabular}{lllll}
\hline $\begin{array}{l}\text { Age } \\
\text { fin 1984 }\end{array}$ & $\begin{array}{l}\text { Intensité } \\
d u \\
\text { dépressage }\end{array}$ & \multicolumn{3}{c}{$\begin{array}{l}\text { Coefficients de corrélation } \\
\text { des variables explicatives }\end{array}$} \\
\cline { 3 - 5 } & & HAUTMOY & HAUTCOR & PCENTCV \\
\hline 4 & faible & 0,3486 & 0,8790 & 0,9313 \\
& fort & 0,5126 & 0,8535 & 0,8753 \\
8 & faible & 0,6622 & 0,8842 & 0,8311 \\
& fort & 0,4923 & 0,9398 & 0,7306 \\
15 & faible & 0,1528 & 0,4599 & 0,4215 \\
& fort & 0,7031 & 0,9178 & 0,9140 \\
20 & faible & 0,8913 & 0,8294 & 0,5242 \\
& fort & 0,5191 & 0,5393 & 0,4351 \\
25 & faible & 0,0856 & 0,8887 & 0,8556 \\
& fort & 0,7163 & 0,9114 & 0,8914 \\
\hline
\end{tabular}

que les rejets sont plus nombreux en 1989 qu'en 1986 dans le cas des éclaircies faible, moyenne et forte, ceci étant contraire à ce qui se passe habituellement en plein découvert. Pour l'éclaircie très forte, il y a le même nombre de rejets en 1986 et 1989 . Les hauteurs moyenne et maximale des rejets sont aussi positivement influencées par l'intensité de l'éclaircie.

Pour caractériser la réaction des cépées partiellement exploitées, on a rapporté le nombre de rejets au nombre et au pourcentage de brins coupés dans la cépée, ainsi qu'à la surface terrière et au pourcentage de surface terrière enlevés dans la cépée. Ces variables ont été traitées par une analyse de variance à 2 facteurs : bloc et traitement.

Les résultats du tableau IV montrent que le nombre de rejets par brin coupé ou par pourcent de brins coupés dans la cépée est influencé positivement et significativement par l'intensité d'éclaircie. Par contre, le nombre de rejets par unité de surface terrière ou par pourcent de surface terrière enlevée dans la cépée n'est pas influencé par l'intensité d'éclaircie. Ces résultats seront repris en détail dans la discussion pour expliquer la réaction de la cépée.

Une autre manière de caractériser la réaction d'une cépée à une exploitation partielle est de se placer à un niveau donné d'éclaircie et de relier la réponse de la cépée (nombre et hauteur des rejets) aux 10 variables qui caractérisent la cépée et le degré d'intervention dans la cépée.

L'analyse des coefficients de corrélations partielles entre les 2 variables expliquées et les 10 variables explicatives montre (tableau $V$ ) que le nombre de rejets est plutôt lié au nombre de brins coupés dans la cépée, tandis que la hauteur des rejets est plutôt liée au pourcentage de 

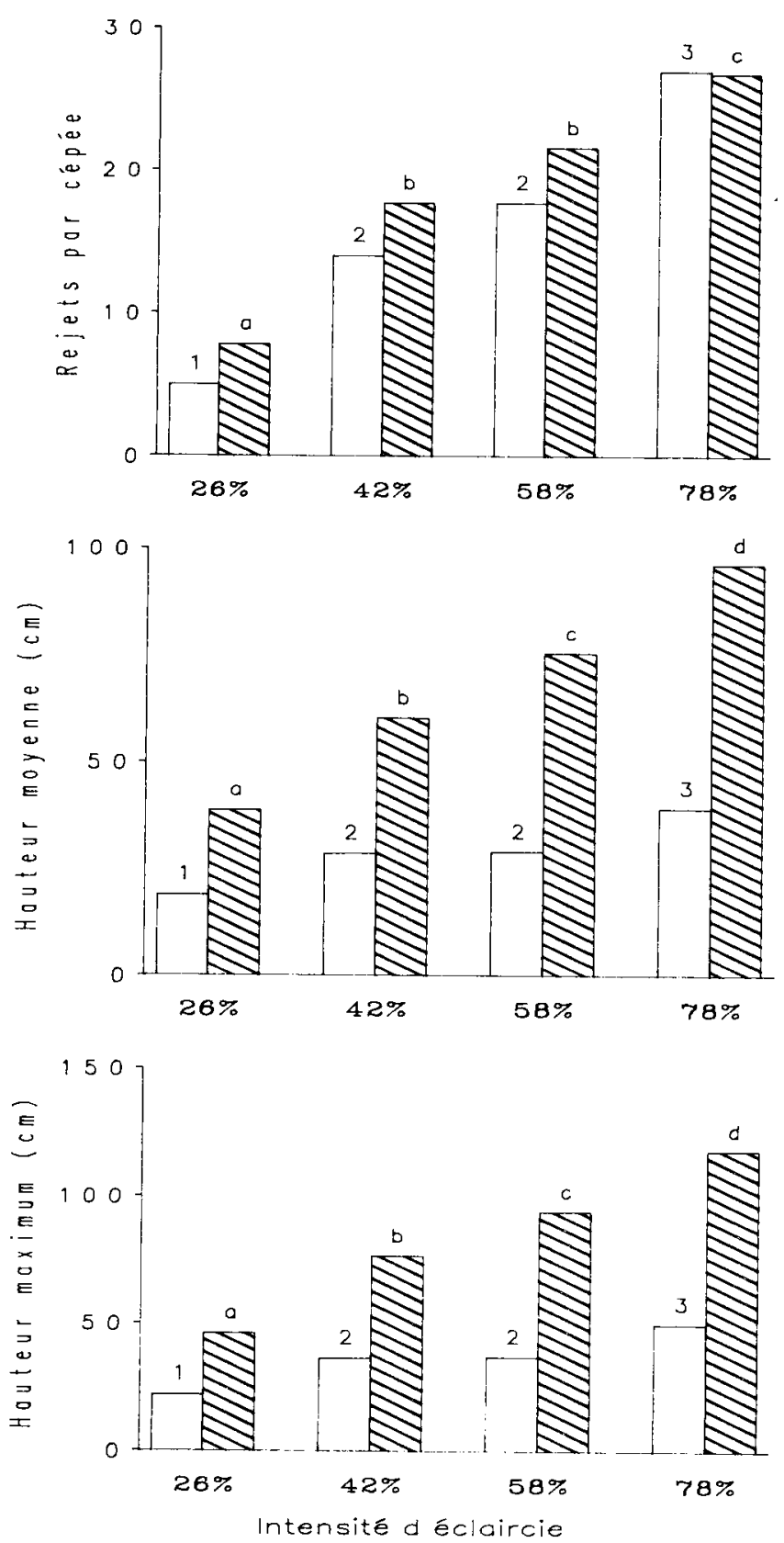

Fig 3. Dénombrement et hauteur des rejets dans les cépées, un an et 4 ans après l'éclaircie des taillis âgés de Puéchabon. Les barres non hachurées et les chiffres concernent les mesures faites fin 1986, les barres hachurées et les lettres celles faites fin 1989. Des chiffres ou des lettres identiques indiquent que l'effet de l'intensité d'éclaircie n'est pas statistiquement significatif au seuil de $5 \%$. 
Tableau IV. Résultats de l'analyse de variance sur les variables synthétiques concernant le nombre de rejets de chaque cépée. Pour chaque variable et pour chaque facteur étudiés, des lettres identiques indiquent des moyennes non statistiquement différentes au seuil de $5 \%$. Les effets bloc ou traitement ou l'interaction sont significatifs au seuil de $1 \%\left(^{* *}\right)$, significatifs au seuil de $5 \%\left(^{*}\right)$ ou non significatifs au seuil de $5 \%$ (NS).

\begin{tabular}{|c|c|c|c|c|}
\hline & $\begin{array}{l}\text { Nb de rejets } \\
\text { par } \\
\text { brin coupé }\end{array}$ & $\begin{array}{l}\text { Nb de rejets } \\
\text { par \% de } \\
\text { brins coupés }\end{array}$ & $\begin{array}{l}\text { Nb de rejets } \\
\text { par surface } \\
\text { terrière } \\
\text { coupée }\end{array}$ & $\begin{array}{l}\text { Nb de rejets } \\
\text { par \% de } \\
\text { surface } \\
\text { terriere coupée }\end{array}$ \\
\hline \multicolumn{5}{|c|}{ Moyenne par bloc } \\
\hline 1 & $6,0^{a}$ & $0,32 a b$ & 0,26 a & $0,64 a$ \\
\hline 2 & $6,0^{a}$ & 0,28 a & $0,17^{b}$ & $0,44^{b}$ \\
\hline 3 & $8,2^{b}$ & $0,34^{b}$ & $0,14^{b}$ & $0,65 a$ \\
\hline \multicolumn{5}{|c|}{ Moyenne par traitement } \\
\hline E faible & $4,2 \mathrm{a}$ & $0,19^{a}$ & $0,16^{a}$ & $0,58^{a}$ \\
\hline E moyenne & 5,5 a & $0,31^{b}$ & $0,20^{a}$ & $0,55 \mathrm{a}$ \\
\hline$E$ forte & $7,5^{b}$ & $0,34^{b}$ & $0,20^{a}$ & $0,49^{a}$ \\
\hline$E$ très forte & $9,1^{c}$ & $0,40^{c}$ & $0,20 \mathrm{a}$ & $0,67^{a}$ \\
\hline $\begin{array}{l}\text { Effet bloc } \\
\text { Effet traitement } \\
\text { Interaction }\end{array}$ & $\begin{array}{l}F=8,10\left(^{* \star}\right) \\
F=17,07\left(^{\star \star}\right) \\
F=1,84(\text { NS })\end{array}$ & $\begin{array}{l}F=3,24\left(^{*}\right) \\
F=14,73\left(^{* *}\right) \\
F=0,48(\mathrm{NS})\end{array}$ & $\begin{array}{l}F=11,22\left({ }^{* *}\right) \\
F=0,68(\mathrm{NS}) \\
F=0,47(\mathrm{NS})\end{array}$ & $\begin{array}{l}F=4,03\left(^{*}\right) \\
F=1,09 \text { (NS) } \\
F=1,34 \text { (NS) }\end{array}$ \\
\hline
\end{tabular}

brins ou de surface terrière enlevés en éclaircie.

\section{DISCUSSION ET CONCLUSION}

Dans la plupart des expérimentations sur l'éclaircie des taillis et des cépées, l'attention est surtout portée sur le comportement des brins sélectionnés (Mer, 1909; Dale et Sonderman, 1984; Aufort, 1985; Dreuillaux et al, 1985). II n'est jamais fait mention des nouveaux rejets qui apparaissent sauf, éventuellement, pour dire qu'il faut les contrôler (Weinstein, 1986). Aussi les résultats que nous avons obtenus sont-ils relativement originaux et nous allons tenter d'en faire une synthèse en examinant successivement l'influence de l'âge du taillis et de l'intensité d'éclaircie qui sont nos 2 principaux facteurs expérimentaux. Nous nous attacherons ensuite à comprendre le fonctionnement de la cépée en réaction à une exploitation partielle.

Nous avons choisi de discuter globalement les 2 expérimentations de La Bruguière et de Puéchabon car les peuplements appartiennent aux mêmes classes de fertilité (Boisserie, 1990). Bien que les interventions sylvicoles n'aient pas été faites la même année, nous avons estimé que cette discussion était possible car nos mesures ne concernent pas une seule année mais l'ensemble des 4 et 5 années qui ont suivi l'exploitation.

\section{Influence de l'âge du taillis}

La réaction à l'éclaircie est variable selon l'âge du taillis. L'extension spatiale de la végétation au sol est plus grande dans les 


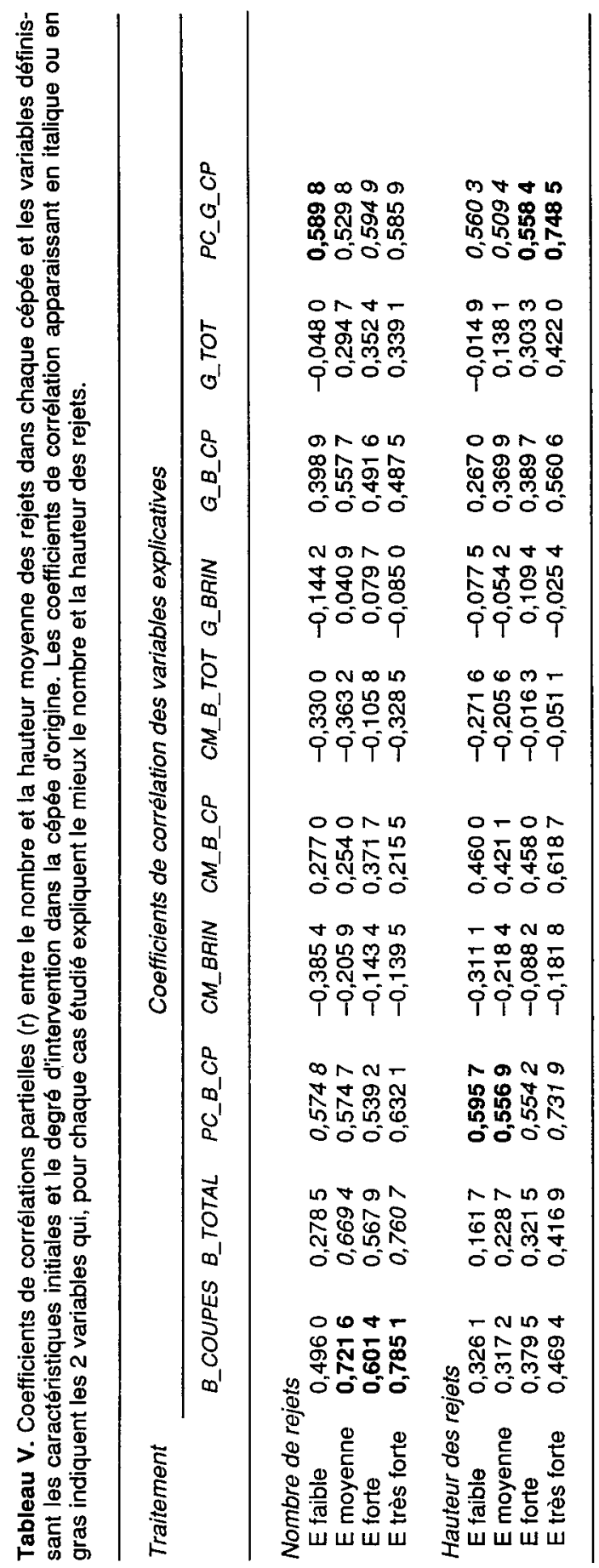


taillis jeunes que dans les taillis âgés à intensité d'éclaircie équivalente. Ceci peut être expliqué par le fait que la densité initiale est supérieure dans les taillis jeunes à celle des taillis âgés et qu'il y a plus de brins coupés en éclaircie dans les taillis jeunes. Comme nous avons vu que le nombre de rejets est lié positivement au nombre de brins coupés, il est normal qu'apparaissent davantage de rejets dans les taillis jeunes, contribuant ainsi à un plus grand recouvrement. Dans les taillis âgés pour lesquels la densité est plus faible en raison de la concurrence intra- et inter-cépées, le recouvrement est moins important. Ce raisonnement fait pour le chêne vert est aussi valable pour les autres espèces arbustives qui sont très nombreuses dans les taillis jeunes et qui ont été progressivement éliminées dans les taillis âgés par la concurrence des cépées de chêne vert.

La biomasse totale des rejets de chêne vert et de l'ensemble de la strate recouvrant le sol augmente régulièrement (bien que de manière non statistiquement significative) avec l'âge des taillis jeunes. Cette constatation nous permet de formuler quelques hypothèses, en tenant compte du fait que les réserves hydrocarbonées de la souche peuvent contribuer à la croissance des nouveaux rejets pendant l'année qui suit le recépage (Pontailler et al, 1984). On peut supposer que si les rejets dans les taillis de 25 ans sont plus vigoureux que ceux des taillis de 4 ans c'est que la souche possède à ce moment-là, davantage de réserves hydrocarbonées mobilisables. Si on extrapole au cas de l'exploitation complète du taillis, il faut donc rechercher des rotations longues : 20-25 ans au minimum, pour assurer la meilleure régénération possible par rejets de souche. II n'est cependant pas possible de fixer une durée optimale, en raison du manque de données expérimentales.
La hauteur moyenne des rejets, mesurée fin 1989 lors des observations de recouvrement a été reliée, pour les 2 interventions les plus fortes, à la hauteur moyenne du taillis préexistant (fig 4). Bien que les rejets des taillis de 43 et 57 ans n'aient que 4 ans - ils grandiront d'environ $10 \mathrm{~cm}$ pendant la $5^{e}$ année - tandis que les autres ont 5 ans, on peut dire qu'à âge égal, leur hauteur est nettement inférieure à celle des rejets des taillis de 4 à 25 ans. On peut interpréter ce résultat comme une baisse de la vigueur des rejets. Ce premier signe visible de l'effet du vieillissement du taillis peut être considéré comme une diminution de la capacité du taillis à rejeter de souche. On voit aussi que, dans les taillis jeunes, la hauteur des jeunes rejets atteint la moitié de la hauteur moyenne du taillis, alors que dans les taillis âgés, elle n'atteint que le quart de la hauteur moyenne du taillis. II y a donc reconstitution d'une strate unique dans les taillis jeunes, alors que la strate arbustive reste séparée de la strate arborescente dans les taillis âgés.

\section{Effet de l'intensité de l'intervention sylvicole}

L'intensité du dépressage ou de l'éclaircie a un effet marqué sur la dynamique d'apparition des nouveaux rejets. Pour les taillis jeunes, les différences entre dépressage faible et dépressage fort, bien que visibles, ne sont pas très importantes en ce qui concerne le recouvrement. Par contre, elles sont beaucoup plus importantes pour la biomasse des rejets de chêne vert qui est 2 fois plus forte dans le dépressage fort que dans le dépressage faible. Ceci est vraisemblablement dû aux différences d'éclairement, le dépressage fort ouvrant beaucoup plus le couvert que le dépressage faible. 


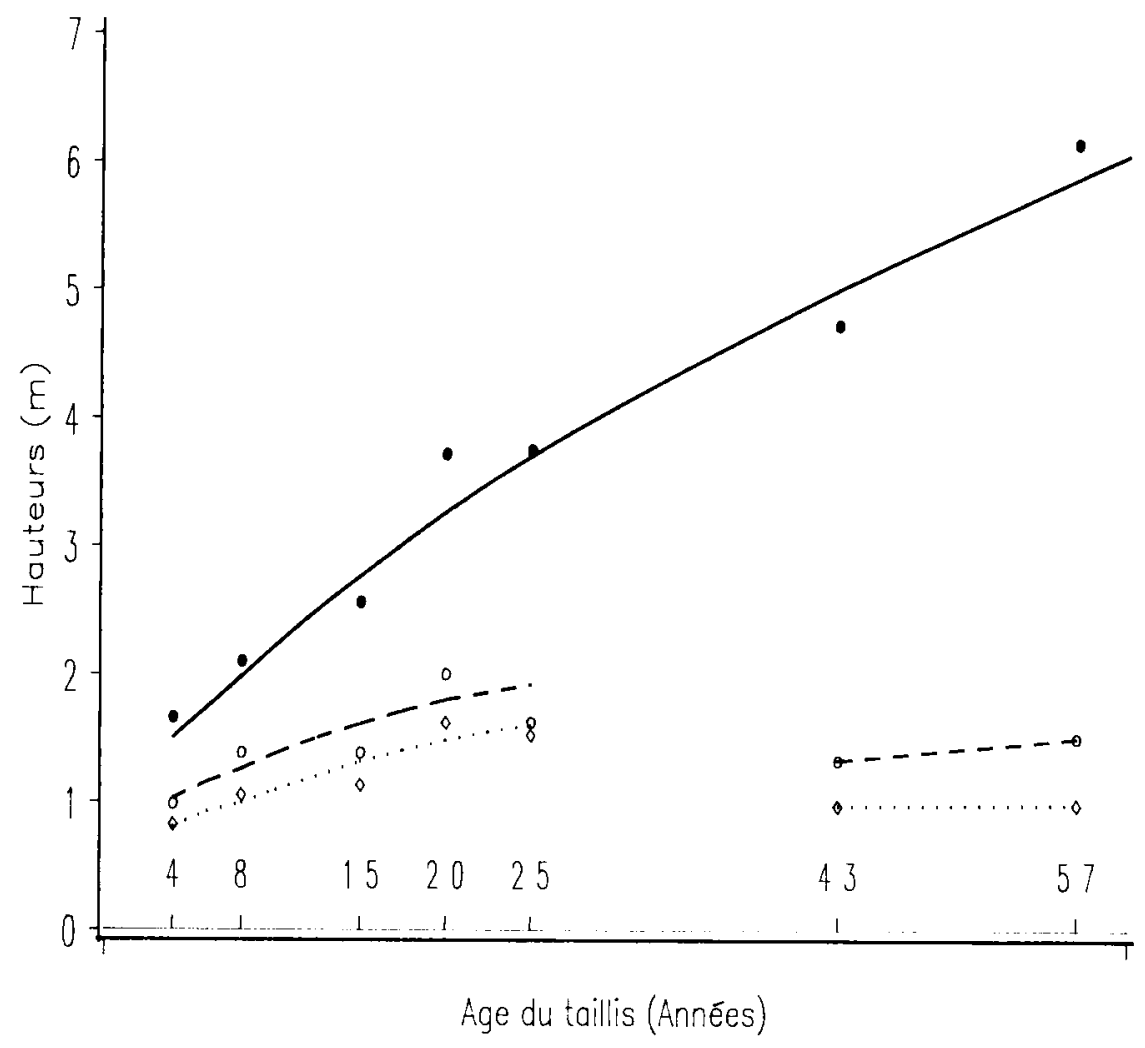

Fig 4. Hauteur moyenne des rejets, fin 1989, dans les taillis fortement dépressés ou très fortement éclaircis (0) et dans les taillis faiblement dépressés ou fortement éclaircis ( $(0)$ en relation avec la hauteur moyenne du taillis initial $(\bullet)$. (Les lignes ont été tracées à main levée).

Dans les taillis âgés, l'intensité de l'éclaircie a un effet positif sur le nombre de rejets émis par les cépées, ainsi que sur la croissance de ces rejets. La croissance en hauteur des rejets paraît très directement liée à l'intensité de l'éclaircie, c'est-à-dire à l'éclairement qui pénètre jusqu'au sol.

On note aussi que, dans les éclaircies faible, moyenne et forte, tous les rejets n'apparaissent pas au cours de la pre- mière année et qu'ils sont plus nombreux au bout de 4 ans. Dans l'éclaircie très forte, le nombre de rejets reste constant. $\mathrm{Si}$ on considère que la mortalité est très faible - pratiquement pas de rejets morts observés lors du deuxième comptage ceci veut dire que seule l'éclaircie très forte apporte toutes les conditions nécessaires à l'entrée en activité de tous les bourgeons proventifs présents à la base des brins dès la première année. Les fac- 
teurs qui peuvent être mis en jeu sont la lumière et la température (Roussel, 1978) ainsi que l'action combinée des principales hormones de croissance : auxines, gibbérellines et cytokinines (Taylor et al, 1982). Dans les $\mathbf{3}$ autres types d'éclaircie, il est probable que les équilibres hormonaux nécessaires à l'activation de tous les bourgeons proventifs n'ont pas été atteints dès la première année.

On remarque aussi que la croissance en hauteur dans l'éclaircie très forte est sensiblement identique à celle obtenue après coupe rase par Floret et al (1986) dans un taillis de chêne voisin et de caractéristiques identiques. Ce résultat est obtenu par la conjonction d'effets antagonistes positifs ou négatifs sur la croissance en hauteur des jeunes rejets soumis à la concurrence des brins de taillis non exploités.

\section{Réaction de la cépée}

Pour aller plus en avant dans l'explication de l'apparition et de la croissance des rejets, il est nécessaire de raisonner au niveau de la cépée tout entière. Le chêne vert se caractérise comme la plupart des autres chênes (Bartet, 1890; Roth et Hepting, 1943) par la présence de nombreux bourgeons d'origine proventive à la base des tiges. Le maintien en repos ou la mise en activité de ces bourgeons proventifs est sous le contrôle des corrélations physiologiques entre les bourgeons et la partie aérienne de la cépée (Champagnat, 1983, 1989). On peut dire qu'à l'intérieur de la cépée, il y a préséance des tiges adultes sur les bourgeons proventifs situés à leur base et que la coupe d'une partie des tiges adultes provoque la mise en activité de tout ou partie des bourgeons proventifs.

Nous allons tenter d'interpréter nos résultats sur l'apparition de nouveaux rejets dans les cépées partiellement exploitées en séparant les facteurs externes liés à l'ouverture du couvert et les facteurs internes liés aux modifications des corrélations entre organes dans la cépée et aux phénomènes hormonaux associés.

Pour comprendre l'effet de l'ambiance environnementale, liée au niveau moyen d'éclaircie, il faut s'affranchir du niveau d'intervention dans la cépée en rapportant le nombre de rejets au degré d'intervention dans la cépée (tableau IV). Nous avons vu que le nombre de rejets rapporté au nombre de brins coupés augmente avec l'intensité de l'éclaircie. Par contre, si le nombre de rejets est rapporté à la grosseur des brins (surface terrière), il reste à peu près constant quelle que soit l'intensité de l'éclaircie. Le premier résultat est dû au fait que, plus l'intensité de l'éclaircie est forte, plus on coupe de grosses tiges produisant davantage de rejets. Par contre, le deuxième résultat prenant en compte la grosseur des tiges coupées indique que, pour un degré d'intervention donné dans la cépée, le nombre de rejets émis est le même quelle que soit l'intensité de l'éclaircie, et donc, que tous les bourgeons proventifs situés à la base des tiges coupées ont été mis en activité.

Pour comprendre l'effet du degré d'intervention dans la cépée, il faut se placer dans des conditions environnementales homogènes donc à un niveau d'éclaircie donné. On a vu alors (tableau V), que le nombre de rejets pour une intensité d'éclaircie donnée est lié positivement au nombre de brins coupés dans la cépée, alors que la hauteur des rejets est liée positivement au pourcentage d'intervention dans la cépée. Pour préciser ces résultats, on a séparé les cépées en cépées entièrement coupées, partiellement coupées ou non coupées, et indiqué le nombre et la hauteur des rejets de 1 an pour chaque intensité d'éclaircie (fig 5). Seuls quelques rejets isolés et de faible croissance apparaissent sur les cépées non coupées tandis que les cépées entièrement coupées 

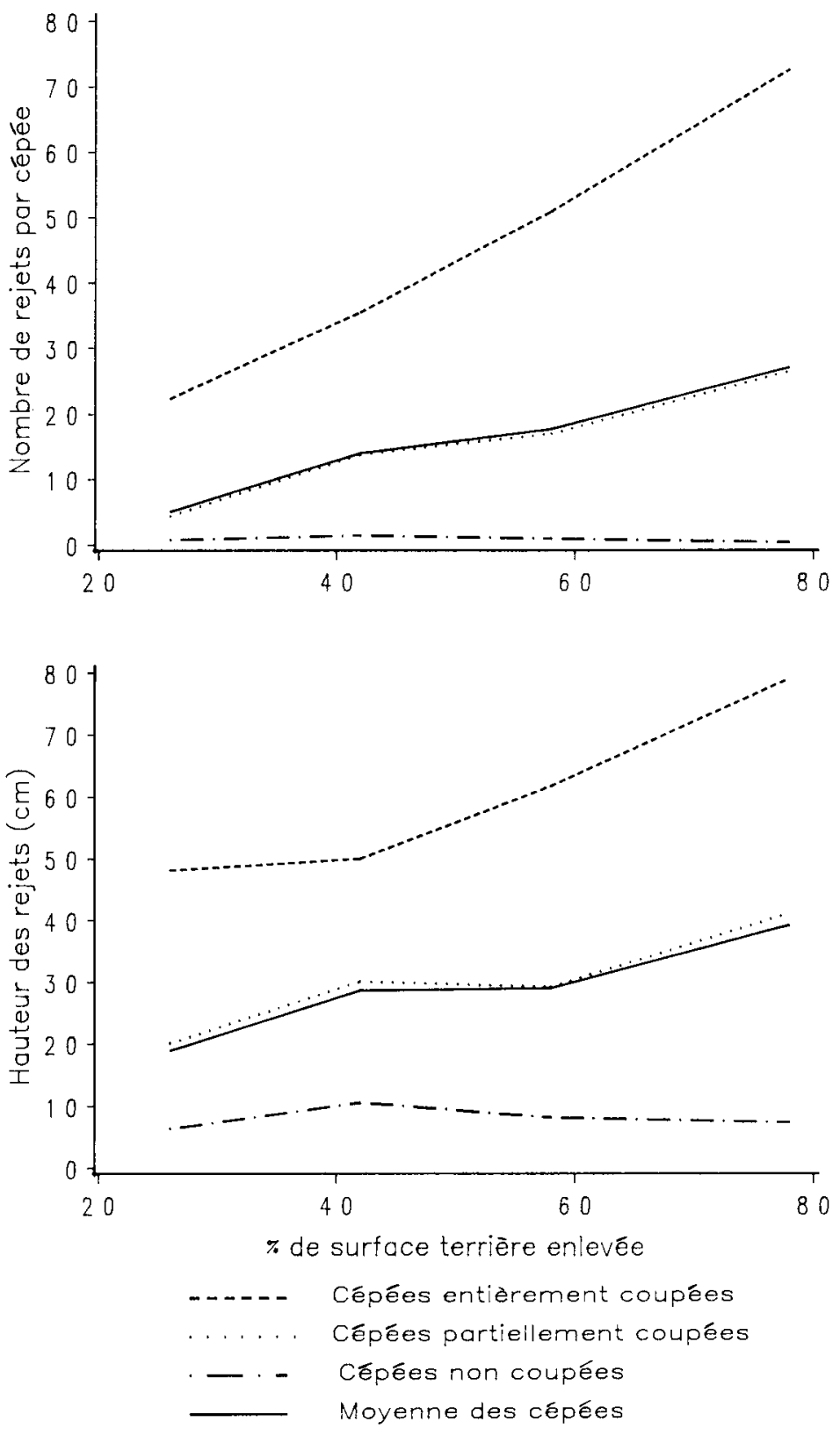

Fig 5. Dénombrement et hauteur des rejets dans les cépées pour les taillis âgés de Puéchabon, fin 1986, en relation avec l'intensité d'éclaircie et le degré de coupe dans la cépée. 
ont un développement d'autant plus important que l'intensité de l'éclaircie est grande.

La discussion précédente ne permet pas de trancher définitivement quant au rôle précis des différents facteurs d'origine interne ou externe qui interviennent dans l'apparition des rejets à la suite d'une coupe partielle dans une cépée.

Nous n'avons pas trouvé d'informations à ce sujet dans la littérature scientifique. On peut cependant raisonner par analogie en analysant les phénomènes mis en jeu lors de l'apparition des gourmands. Les gourmands sont issus de bourgeons épicormiques de même nature que les bourgeons proventifs produisant les rejets de souche. En dehors du fait que les gourmands apparaissent sans levée préalable de la préséance de l'arbre mère, le fonctionnement de ces 2 types de bourgeons est le même.

La plupart des auteurs (Ward, 1966; Dale et Sonderman, 1984; Wignall et Browning, 1988; Harmer, 1988) pensent à l'action combinée de la lumière, voire de la température et des facteurs hormonaux. II est certain que, sans jouer un rôle moteur dans la mise en activité des bourgeons épicormiques, la lumière est le facteur qui, dans la plupart des cas, déclenche le processus. On parle habituellement de seuil d'éclairement au-dessus duquel les bourgeons sont mis en activité. Nous pensons qu'il s'agit plus sûrement d'une variation de l'éclairement par rapport au niveau ambiant moyen et plus généralement d'une variation des taux d'hormones par rapport aux niveaux préexistants qui provoquent l'activation des bourgeons.

Il est vrai que des rejets de souche peuvent apparaître à la base de cépées intactes. Mais dans la plupart des cas, ceci se produit à la suite de traumatismes subis par la cépée : bris de branches, rameaux desséchés à la suite d'attaques d'insectes (Coraebus bifasciatus) qui, sans provoquer de modifications sensibles de l'environnement, sont suffisants pour modifier les corrélations entre organes.

En raison de la complexité des phénomènes mis en jeu pour expliquer la mise en activité des bourgeons proventifs, il est difficile de les séparer. II faudrait pouvoir expérimenter en contrôlant plus précisément les facteurs du milieu, tout en restant dans les conditions naturelles, ce que nous n'avons pas eu la possibilité de faire.

\section{Conséquences sylvicoles}

Les conséquences sylvicoles de notre expérimentation sont très importantes. Au cours d'une précédente étude, nous avons vu (Ducrey et Toth, 1992) que les dépressages et les éclaircies favorisent la croissance en circonférence des brins sélectionnés quand on élimine plus de $50 \%$ de la surface terrière initiale. Mais le développement des rejets à la suite de ces interventions sylvicoles est encore plus dynamique et le nombre et la hauteur de ces jeunes rejets augmentent proportionnellement à la surface terrière exploitée. II en résulte qu'une ouverture du couvert faible, dans les éclaircies faibles ou moyennes, provoque l'apparition de rejets et d'une strate arbustive au sol importante, sans gain de croissance pour le taillis. Ce gain de croissance n'est rencontré que dans les éclaircies fortes et très fortes, mais dans ce cas, la strate arbustive au sol devient encore plus abondante.

II devient alors nécessaire de la contrôler en utilisant des moyens adaptés aux buts recherchés. Le contrôle des rejets dans le cas de coupures de combustibles doit être fait vigoureusement par recépage ou broyage répété des rejets, ou par l'utilisation intensive du pâturage caprin. Dans le cas de la conversion en futaie sur souche, et si on ne recherche pas un gain 
de productivité rapide, on veillera essentiellement à ne pas reconstituer un «taillis dans le taillis". On pourra agir beaucoup plus prudemment en faisant de petites éclaircies répétées pour diminuer progressivement la densité du taillis, mais suffisamment espacées pour que le couvert puisse se reconstituer entre 2 éclaircies.

\section{REMERCIEMENTS}

Les auteurs remercient tout particulièrement Messieurs Toth et Turrel, ainsi que l'équipe technique de la Station de sylviculture méditerranéenne pour leur aide précieuse tout au long de cette étude.

\section{RÉFÉRENCES}

Auclair D (1986) Terminologie des taillis. In: Compte rendu de la réunion du Groupe Taillis, 20-21 mars 1986, Univ Orsay, 66-68

Aufort JM (1985) Influence d'une éclaircie sur la croissance des rejets au cours de leur $2^{\mathrm{e}}$ et $3^{e}$ année de végétation. In: Compte rendu de la réunion du Groupe Taillis, 14-15 mars 1985, Univ Limoges, 90-103

Bartet E (1890) Influence exercée par l'époque de l'abattage sur la production et le développement des rejets des souches dans les taillis. Rev Eaux For 29, 310-313

Boisserie M (1990) Influence des traitements sylvicoles (exploitation à blanc, dépressage, éclaircies) sur la repousse et la croissance des taillis de chêne vert. Mémoire de $3^{\theta}$ année ENITEF, INRA Avignon, $72 \mathrm{p}+$ annexes

Champagnat $P$ (1983) Quelques réflexions sur la dormance des bourgeons des végétaux ligneux. Physiol Vég 21, 607-618

Champagnat $P$ (1989) Rest and activity in vegetative buds of trees. Ann Sci For 46 suppl, 9s-26s
Dale ME, Sonderman DL (1984) Effect of thinning on growth and potential quality of young White oak crop trees. US Dep Agric For Serv, Northeastern For Exp Stn, Res Paper NE-539, $12 \mathrm{p}$

Dreuillaux JM, Legay B, Pontailler JY (1985) Bilan d'un dépressage précoce sur taillis de Châtaignier. In: Compte rendu de la réunion du Groupe Taillis, 14-15 mars 1985, Univ Limoges, 85-86

Ducrey M (1988) Sylviculture des taillis de chëne vert. Pratiques traditionnelles et problématique des recherches récentes. Rev For Fr XL, 302-313

Ducrey M, Toth J (1992) Effect of cleaning and thinning on height growth and girth increment in holm oak coppices (Quercus ilex L). Vegetatio (in press)

Ducrey M, Toth J, Turrel M (1986) Protocole d'étude du dépressage dans des taillis de chêne vert âgés de 4 à 25 ans en vue de leur conversion en futaie sur souche. Forêt communale de La Bruguiere, Gard. INRA Avignon. Stn Sylvic Méditerr. Doc int $n^{\circ} 19-$ 86, $26 \mathrm{p}$

Ducrey M, Toth J, Turrel M (1987) Protocole d'étude des éclaircies dans des taillis de chêne vert âgés de 43 et 57 ans, en vue de leur conversion en futaie sur souche (Forêt domaniale de Puéchabon, Hérault). INRA Avignon, Stn Sylvic Méditerr Doc Int $n^{\circ} 4-$ $87,23 p$

Fink VS (1983) The occurrence of adventitious and proventitious buds within the bark of some temperate and tropical trees. Am J Bot 70, 532-542

Floret C, Galan MJ, Le Floc'h E, Romane F (1986) Croissance d'un jeune taillis de chêne vert; quelques remarques sur les résultats de 3 ans d'observations dans la station de Puéchabon. In: Compte rendu de la réunion du Groupe Taillis, 20-21 mars 1986, Univ Orsay, 38-40

Jolyet A (1916) Traité pratique de sylviculture. Baillière, Paris, $724 p$

Harmer R (1988) Production and use of epicormic shoots for the vegetative propagation of mature Oak. Forestry 61, 305-316 
Kauppi A, Rinne P, Ferm A (1987) Initiation structure and sprouting of dormant basal buds in Betula pubescens. Flora 179, 55-83

Kormanik PP, Brown CL (1973) Vegetative propagation of some selected hardwood forest species in the Southeastern United States. NZJ For Sci 4, 228-234

Mathey A (1929) Traité théorique et pratique des taillis. Vilaire, Le Mans, $353 \mathrm{p}$

Mer E (1909) Influence de l'éclaircie des cépées sur la croissance en grosseur des rejets réservés. Rev Eaux For 48, 492-497, 524-535, 551-562

Perrin H (1964) Sylviculture. Tome II. Le traitement des forêts. Théorie et pratique des techniques sylvicoles. Ec Nat Eaux For, Nancy, $411 p$

Piskoric O (1963) The dynamics of height increment of coppice shoots of Evergreen Oak. Sumarski List 87 (3-4), 122-133

Pontailler JY, Leroux M, Saugier B (1984) Évolution d'un taillis de châtaignier après coupe rase : photosynthèse et croissance des rejets. Acta $C_{\text {Ecol }}$ EEcol Plant 19, 89-99
Roth ER, Hepting GH (1943) Origin and development of the oak stump sprouts as affecting their likelihood to decay. J For 41, 2736

Roussel L (1978) Lumière, gourmands et rejets de souche. Rev For Fr XXX, 533-550

Seigue A (1985) La forêt circum-méditerranéenne et ses problèmes. Maisonneuve et Larose, Paris, $502 \mathrm{p}$

Taylor JS, Blake TJ, Pharis RP (1982) The role of hormones and carbohydrates in the growth and survival of coppiced Eucalyptus seedlings. Physiol Plant 55, 421-430

Ward WW (1966) Epicormic branching of Black and White oak. For Sci 12, 290-296

Weinstein A (1986) The influence of silvicultural treatments on tree response and on the live ground cover in an east mediterranean oak scrub community. For Mediterr VIII, 127-132

Wignall TA, Browning C (1988) The effects of stand thinning and artificial shading on epicormic bud emergence in Pedonculate oak (Quercus robur L). Forestry 61, 45-59 